\title{
Prognosis After Resection of Barcelona Clinic Liver Cancer (BCLC) Stage 0, A, and B Hepatocellular Carcinoma: A Comprehensive Assessment of the Current BCLC Classification
}

Diamantis I. Tsilimigras, MD ${ }^{1}$, Fabio Bagante, $\mathrm{MD}^{1,2}$, Kota Sahara, $\mathrm{MD}^{1}$, Dimitrios Moris, MD, $\mathrm{PhD}^{1}$, J. Madison Hyer, MS ${ }^{1}$, Lu Wu, MD ${ }^{1}$, Francesca Ratti, $\mathrm{MD}^{3}$, Hugo P. Marques, $\mathrm{MD}^{4}$, Olivier Soubrane, $\mathrm{MD}^{5}$, Anghela Z. Paredes, MD, MS ${ }^{1}$, Vincent Lam, $\mathrm{MD}^{6}$, George A. Poultsides, $\mathrm{MD}^{7}$, Irinel Popescu, $\mathrm{MD}^{8}$, Sorin Alexandrescu, $\mathrm{MD}^{8}$, Guillaume Martel, $\mathrm{MD}^{9}$, Aklile Workneh, $\mathrm{MD}^{9}$, Alfredo Guglielmi, $\mathrm{MD}^{2}$, Tom Hugh, $\mathrm{MD}^{10}$, Luca Aldrighetti, $\mathrm{MD}^{3}$, Itaru Endo, MD, PhD ${ }^{11}$, and Timothy M. Pawlik, MD, MPH, PhD, FACS $^{1}$

\begin{abstract}
${ }^{1}$ Department of Surgery, The Ohio State University Wexner Medical Center, Columbus, OH; ${ }^{2}$ Department of Surgery, University of Verona, Verona, Italy; ${ }^{3}$ Department of Surgery, Ospedale San Raffaele, Milan, Italy; ${ }^{4}$ Department of Surgery, Curry Cabral Hospital, Lisbon, Portugal; ${ }^{5}$ Department of Hepatobiliopancreatic Surgery, APHP, Beaujon Hospital, Clichy, France; ${ }^{6}$ Department of Surgery, Westmead Hospital, Sydney, NSW, Australia; ${ }^{7}$ Department of Surgery, Stanford University, Stanford, CA; ${ }^{8}$ Department of Surgery, Fundeni Clinical Institute, Bucharest, Romania; ${ }^{9}$ Department of Surgery, University of Ottawa, Ottawa, ON, Canada; ${ }^{10}$ Department of Surgery, The University of Sydney, School of Medicine, Sydney, NSW, Australia; ${ }^{11}$ Yokohama City University School of Medicine, Yokohama, Japan
\end{abstract}

\begin{abstract}
Background. Although the Barcelona Clinic Liver Cancer (BCLC) staging system has been largely adopted in clinical practice, recent studies have questioned the prognostic stratification of this classification schema, as well as the proposed treatment allocation of patients with a single large tumor.

Methods. Patients who underwent curative-intent hepatectomy for histologically proven hepatocellular carcinoma (HCC) between 1998 and 2017 were identified using an international multi-institutional database. Overall survival (OS) among patients with BCLC stage 0, A, and B was
\end{abstract}

Electronic supplementary material The online version of this article (https://doi.org/10.1245/s10434-019-07580-9) contains supplementary material, which is available to authorized users.

(C) Society of Surgical Oncology 2019

First Received: 3 March 2019;

Published Online: 2 July 2019

T. M. Pawlik, MD, MPH, PhD, FACS

e-mail: tim.pawlik@osumc.edu examined. Patients with a single large tumor were classified as BCLC stage A1 and were independently assessed.

Results. Among 814 patients, 68 (8.4\%) were BCLC-0, $310(38.1 \%)$ were BCLC-A, 279 (34.3\%) were BCLC-A1, and 157 (19.3\%) were BCLC-B. Five-year OS among patients with BCLC stage 0, A, A1, and B HCC was $86.2 \%, \quad 69.0 \%, 56.9 \%$, and $49.9 \%$, respectively $(p<0.001)$. Among patients with very early- and earlystage HCC (BCLC 0, A, and A1), patients with BCLC stage A1 had the worst OS $(p=0.0016)$. No difference in survival was noted among patients undergoing surgery for BCLC stage A1 and B HCC (5-year OS: $56.9 \%$ vs. $49.9 \%$; $p=0.259$ ) even after adjusting for competing factors (hazard ratio $0.83,95 \%$ confidence interval $0.54-1.28$; $p=0.40$ ).

Conclusion. Prognosis following liver resection among patients with BCLC-A1 HCC was similar to patients presenting with BCLC-B tumors. Surgery provided acceptable long-term outcomes among select patients with BCLC-B HCC. Designation into BCLC stage B should not be considered an a priori contraindication to surgery. 
Hepatocellular carcinoma (HCC) accounts for over 70\% of primary liver malignancies and has had an increased incidence and cancer-related mortality over the past 2 decades. ${ }^{1}$ Surgery, in the form of resection, ablation or liver transplantation, remains the mainstay of treatment for patients with resectable disease. ${ }^{2,3}$ Several staging systems have been proposed to define the prognosis of patients with $\mathrm{HCC}$, as well as inform treatment recommendations. ${ }^{4,5}$ The Barcelona Clinic Liver Cancer (BCLC) system is unique in that it seeks to determine patient prognosis, as well as recommends specific treatment algorithms based on HCC tumor stage. ${ }^{6}$ To this point, the BCLC staging system recommends that only patients with very early- and earlystage (BCLC stage 0 and A, respectively) HCC should undergo surgical resection. In contrast, patients with intermediate (BCLC stage B) and advanced (BCLC stage C) stage $\mathrm{HCC}$ are recommended to receive transarterial chemoembolization (TACE) or sorafenib, respectively. ${ }^{2,6}$

In 2011, the original BCLC system was updated to designate a single large $\mathrm{HCC}(\geq 5 \mathrm{~cm})$ as BCLC stage A rather than stage $\mathrm{B}^{7}$ The revised BCLC classification schema has been endorsed by the European Association for the Study of the Liver (EASL), ${ }^{2}$ as well as the American Association for the Study of Liver Diseases (AASLD). ${ }^{3}$ Nevertheless, increasing tumor size has been associated with a higher incidence of microvascular invasion, a known risk factor for recurrence. ${ }^{8,9}$ As such, several investigators have questioned the classification of single large tumor as BCLC stage $A{ }^{10,11}$ In turn, the prognostic discrimination of the revised BCLC classification has come into question. In addition, some surgeons have advocated for liver resection in select patients with intermediate (BCLC stage B) or even advanced (BCLC stage C) stage HCC, which is beyond the current BCLC treatment guidelines. ${ }^{12}$ For example, in a report of 85 patients with multinodular BCLC stage B HCC, Wada et al. reported a 5-year overall survival (OS) of $63.4 \%$ and concluded that designation as BCLC stage B should not be an absolute contraindication to surgery. ${ }^{12}$ Given the debate as to whether the revised BCLC system performs well in terms of prognostic stratification and treatment allocation for patients with various stages of HCC, we sought to characterize the outcomes of patients undergoing liver resection for BCLC stage 0 , A, and B HCC. Specifically, the objective of the current study was to determine whether the updated BCLC staging system performed well in stratifying patients with HCC relative to long-term prognosis, with a particular focus on the differences among patients with a single large HCC (i.e. stage A) versus stage B HCC.

\section{MATERIALS AND METHODS}

\section{Study Population and Data Collection}

Patients who underwent resection of HCC between 1998 and 2017 were identified from an international multi-institutional database. Patients were treated at one of the 11 participating institutions: The Ohio State University Wexner Medical Center, Columbus, OH, USA; Yokohama City University School of Medicine, Yokohama, Japan; University of Verona, Verona, Italy; Ospedale San Raffaele, Milano, Italy; Curry Cabral Hospital, Lisbon, Portugal; APHP, Beaujon Hospital, Clichy, France; Westmead Hospital, Sydney, NSW, Australia; Stanford University, Stanford, CA, USA; Fundeni Clinical Institute, Bucharest, Romania; University of Ottawa, Ottawa, ON, Canada; and The University of Sydney, School of Medicine, Sydney, NSW, Australia. Patients were followed and outcomes were recorded in a prospectively maintained multi-institutional database. Patients with very early (BCLC stage 0), early (BCLC stage A) or intermediate (BCLC stage B) stage $\mathrm{HCC}$, according to the latest BCLC staging system, ${ }^{2}$ were included in the analytic cohort. Patients with advanced stage (BCLC stage C) HCC and individuals without follow-up data were excluded from the analysis. The study was approved by the Institutional Review Boards of all participating institutions.

\section{Variables and Outcomes of Interest}

The primary outcome was OS, which was defined as the time interval between the date of hepatectomy and the date of death or last follow-up. The primary independent variable was the BCLC stage as defined by the latest EASL guidelines. ${ }^{2}$ In brief, BCLC 0 was defined as a single tumor $<2 \mathrm{~cm}$; BCLC A was defined as a single tumor $2-5 \mathrm{~cm}$ or two to three nodules, all $<3 \mathrm{~cm}$; and BCLC B referred to two to three nodules $\geq 3 \mathrm{~cm}$ or $\geq 4$ nodules. For the purpose of this study, patients with a single large HCC $(\geq 5 \mathrm{~cm})$ were subclassified as BCLC stage A1 (Fig. 1).

Demographic and clinical data included age, sex, American Society of Anesthesiologists (ASA) score, Charlson Comorbidity Index score (CCS), history of cirrhosis, hepatitis B virus (HBV)/hepatitis C virus (HCV) infection, ascites within 30 days prior to surgery, body mass index (BMI), laboratory values (i.e. $\alpha$-fetoprotein [AFP], platelet count [PLT], albumin, total bilirubin, alanine aminotransferase [ALT], aspartate aminotransferase [AST], prothrombin time [PT]), Child-Pugh liver function, BCLC classification, type of surgical resection (i.e. major or minor), minimally invasive surgery, tumor grade, tumor size, pathologic lymphovascular invasion and liver capsule 

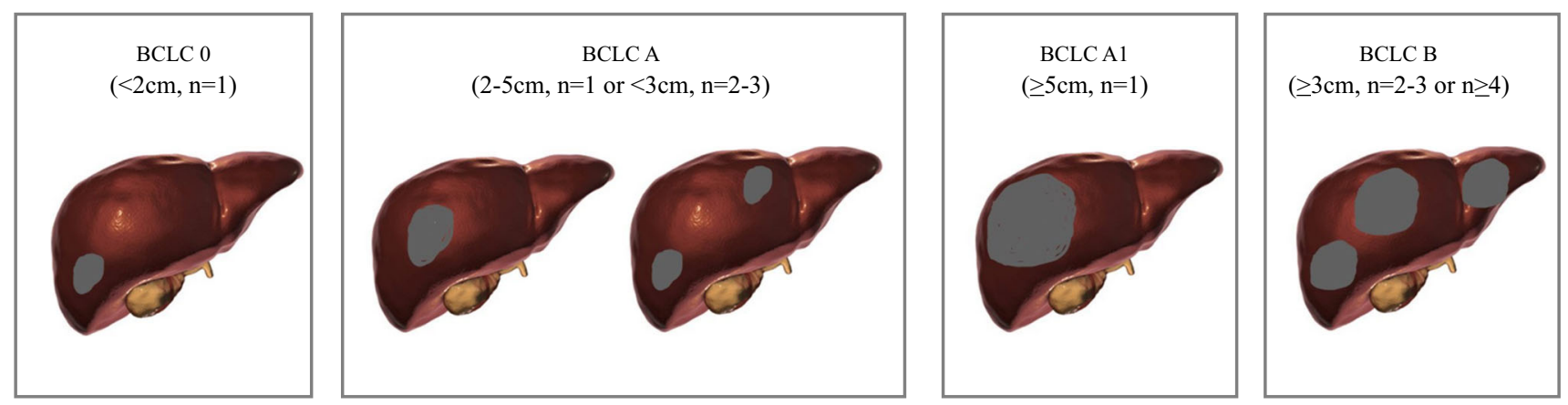

FIG. 1 Schematic presentation of BCLC stage 0, A, A1, and B hepatocellular carcinoma. BCLC Barcelona Clinic Liver Cancer

involvement, as well as margin status (i.e. R0, R1, or R2). Major hepatectomy was defined as resection of three or more Couinaud segments.

\section{Statistical Analysis}

Descriptive statistics were presented as median (interquartile range $[\mathrm{IQR}]$ ) and frequency (\%) for continuous and categorical variables, respectively. Bivariate survival analyses were performed using the log-rank test and were presented using Kaplan-Meier curves. Multivariable survival analyses were performed using Cox proportional hazards regression analysis. The level of statistical significance was set at $\alpha=0.05$. All analyses were performed using STATA version 12.0 (StataCorp LLC, College Station, TX, USA).

\section{RESULTS}

\section{Patient and Tumor Characteristics}

The analytic cohort was comprised of 814 patients with HCC who underwent surgical resection and met the inclusion criteria. Overall, patients were categorized into BCLC $0 \quad(n=68,8.4 \%)$, BCLC A $(n=310,38.1 \%)$, BCLC A1 $(n=279,34.3 \%)$, and BCLC B $(n=157$, $19.3 \%$ ) stage (Fig. 1). Among the entire cohort, median patient age was 68 years (IQR 60-74), most patients were male $(n=628,77.3 \%)$, and had an ASA score of $>2$ $(n=457,57.7 \%)$ and CCS of $>3 \quad(n=448,55 \%)$ (Table 1). A history of cirrhosis, as well as HBV and HCV infection was present in $37.6 \% \quad(n=306), 16.5 \%$ $(n=134)$, and $31.4 \%(n=253)$ of patients, respectively. Only a minority of individuals had ascites prior to surgery ( $n=28,3.4 \%$ ). Median tumor size was $5 \mathrm{~cm}$ (IQR 3-9) and most tumors were well/moderately differentiated $(n=646,80.8 \%)$. Approximately one-quarter of patients underwent minimally invasive surgery $(n=205,25.2 \%)$; approximately one-third had a major resection $(n=290$, $36.1 \%$ ). The vast majority of patients had R0 HCC resection $(n=658,81.6 \%)$. On histological examination, $42.7 \%(n=336)$ of patients had lymphovascular invasion, whereas liver capsule involvement was present in $36.6 \%$ $(n=230)$ of patients. Median follow-up was 29.5 months (IQR 14.5-51.1).

\section{Survival Analysis: Barcelona Clinic Liver Cancer} (BCLC) Stage 0, A, A1, and B Hepatocellular Carcinoma $($ HCC)

Several variables were associated with prognosis after HCC resection (Table 2). Of note, survival was associated with BCLC staging classification; 5-year OS among patients with BCLC stage $0, \mathrm{~A}, \mathrm{~A} 1$, and B HCC was $86.2 \%$ (95\% confidence interval [CI] 66.2-94.8), 69.0\% (95\% CI 60.8-75.8), 56.9\% (95\% CI 47.5-65.3), and 49.9\% (95\% CI 37.8-60.9), respectively $(p<0.001)$. Among patients with very early- and early-stage HCC (BCLC 0, A, and A1), patients with BCLC stage A1 (single tumors $\geq 5 \mathrm{~cm}$ ) had the worst OS ( $p=0.0016$ ) (Fig. 2a). Perhaps of more interest, there was no difference in survival between patients undergoing surgery for BCLC stage A1 and stage B HCC (3-year OS: $71.7 \%$ vs. $65.5 \%$; 5-year OS: $56.9 \%$ vs. $49.9 \% ; p=0.259$ ) (Fig. $2 b$ ).

\section{Survival Analysis: Other factors}

Apart from BCLC stage, several other factors were associated with survival (Table 2). Of note, patients with AFP $\leq 400 \mathrm{ng} / \mathrm{mL}$ had a 5 -year OS of $65.7 \%(95 \%$ CI 59.6-71.1) versus $53.5 \%$ (95\% CI 43.1-62.8) among patients with AFP $>400 \mathrm{ng} / \mathrm{mL}(p<0.001)$. In addition, 5-year OS decreased from $67.1 \%$ (95\% CI 61.2-72.3) among patients with well/moderately differentiated tumors to $44.0 \%$ (95\% CI 32.6-54.7) for poorly/undifferentiated tumors $(p<0.001)$. Patients undergoing major resection had a 5-year OS of 57.2\% (95\% CI 48.6-64.9) versus $66.1 \%$ (95\% CI 59.3-72.0) among individuals who underwent a minor resection $(p=0.008)$. Perhaps not surprisingly, patients with microscopic lymphovascular 
TABLE 1 Demographics and patient characteristics in the entire cohort $(N=814)$

\begin{tabular}{l} 
Variable \\
\hline Age, years [median (IQR)] \\
$\leq 65$ \\
$>65$ \\
Sex \\
Male \\
Female \\
ASA-PS \\
$\leq 2$ \\
$>2$
\end{tabular}

Charlson comorbidity index score

$$
\begin{aligned}
& \leq 3 \\
& >3
\end{aligned}
$$

Cirrhosis

No

Yes

HBV infection

No

Yes

HCV infection

No

Yes

Ascites within 30 days prior to surgery

No

Yes

BMI, $\mathrm{kg} / \mathrm{m}^{2}$

$<17.5$

17.5-30

$\geq 30$

Platelet count, $\times 10^{3} / \mu \mathrm{L}$

$\leq 150$

$>150$

Albumin, g/dL

$$
\leq 3.5
$$

$>3.5$

Total bilirubin, $\mathrm{mg} / \mathrm{dL}$

$$
\begin{aligned}
& \leq 1.2 \\
& >1.2
\end{aligned}
$$

AST, U/L

$$
\leq 40
$$$$
>40
$$

ALT, U/L

$$
\begin{aligned}
& \leq 56 \\
& >56
\end{aligned}
$$

PT/INR

$$
\leq 1.1
$$$$
>1.1
$$

497 (61.1)

628 (77.3)

184 (22.7)

335 (42.3)

457 (57.7)

366 (45.0)

448 (55.0)

508 (62.4)

306 (37.6)

$680(83.5)$

134 (16.5)

552 (68.6)

253 (31.4)

786 (96.6)

28 (3.4)

15 (1.8)

$566(69.5)$

233 (28.6)

253 (31.1)

$561(68.9)$

174 (21.4)

640 (78.6)

732 (89.9)

$82(10.1)$

334 (41.6)

469 (58.4)

$528(65.4)$

$280(34.7)$

629 (77.3)

185 (22.7)
TABLE 1 continued

Variable

AFP, ng/mL

$$
\leq 400
$$

$581(71.4)$

$>400$

233 (28.6)

Child-Pugh classification

$$
\text { A }
$$

780 (95.8)

$$
\text { B }
$$

BCLC staging classification$$
0
$$

A

A1

$310(38.1)$

279 (34.3)

B

157 (19.3)

Minimally invasive surgery

No

607 (74.8)

Yes

205 (25.2)

Type of resection

Minor

514 (63.9)

Major

Tumor size, cm [median (IQR)]

290 (36.1)

Grade

Well to moderate

5 (3-9)

Poor to undifferentiated

646 (80.8)

154 (19.3)

Lymphovascular invasion

No

451 (57.3)

Yes

336 (42.7)

Liver capsule involvement

No

399 (63.4)

Yes

230 (36.6)

Margin status

R0

658 (81.6)

R1

129 (16.0) invasion $(p<0.001)$, liver capsule involvement $(p<0.001)$, or R1/R2 $(p=0.004)$ had worse 5-year OS than their respective counterparts.

\section{Multivariable Survival Analysis}

On multivariable analysis, compared with patients with BCLC stage B HCC, patients with BCLC stage 0 (hazard 
TABLE 2 Factors associated with 3- and 5-year survival in the entire cohort $(N=814)$

\begin{tabular}{|c|c|c|c|c|c|}
\hline Variable & 3 -year OS (\%) & $95 \% \mathrm{CI}$ & 5 -year OS (\%) & $95 \% \mathrm{CI}$ & $p$ value \\
\hline Age, years & & & & & 0.480 \\
\hline$\leq 65$ & 76.8 & $70.7-81.8$ & 63.7 & $55.5-70.8$ & \\
\hline$>65$ & 74.8 & $69.6-79.2$ & 61.8 & $54.9-67.9$ & \\
\hline Sex & & & & & 0.487 \\
\hline Male & 74.9 & $70.4-78.7$ & 61.3 & $55.2-66.8$ & \\
\hline Female & 78.0 & $69.6-84.4$ & 66.2 & $55.2-75.0$ & \\
\hline ASA-PS & & & & & 0.430 \\
\hline$\leq 2$ & 77.7 & $71.4-82.7$ & 62.8 & $53.5-70.7$ & \\
\hline$>2$ & 75.2 & $70.0-79.5$ & 64.0 & $57.3-70.0$ & \\
\hline Charlson comorbidity index score & & & & & 0.102 \\
\hline$\leq 3$ & 75.9 & $70.5-80.5$ & 68.2 & $61.8-73.8$ & \\
\hline$>3$ & 75.2 & $69.5-80.1$ & 53.5 & $44.4-61.7$ & \\
\hline Cirrhosis & & & & & 0.387 \\
\hline No & 77.6 & $71.3-82.7$ & 57.9 & $48.3-66.3$ & \\
\hline Yes & 74.4 & $69.4-78.7$ & 64.8 & $58.6-70.4$ & \\
\hline HBV infection & & & & & 0.302 \\
\hline No & 75.2 & $70.9-78.9$ & 60.5 & $54.6-65.9$ & \\
\hline Yes & 77.8 & $68.1-84.9$ & 70.9 & $59.6-79.6$ & \\
\hline HCV infection & & & & & 0.653 \\
\hline No & 73.5 & $68.7-77.8$ & 62.6 & $56.3-68.2$ & \\
\hline Yes & 79.7 & $73.0-84.9$ & 62.3 & $52.2-70.8$ & \\
\hline AFP, ng/mL & & & & & $<0.001$ \\
\hline$\leq 400$ & 78.7 & $74.3-82.4$ & 65.7 & $59.6-71.1$ & \\
\hline$>400$ & 67.3 & $59.1-74.2$ & 53.5 & $43.1-62.8$ & \\
\hline Child-Pugh classification & & & & & 0.987 \\
\hline A & 75.6 & $71.7-79.1$ & 62.9 & $57.6-67.7$ & \\
\hline B & 75.4 & $49.5-89.3$ & 52.8 & $20.9-77.0$ & \\
\hline BCLC staging classification & & & & & $<0.001$ \\
\hline 0 & 91.9 & $79.6-97.0$ & 86.2 & $66.2-94.8$ & \\
\hline A & 80.5 & $74.4-85.3$ & 69.0 & $60.8-75.8$ & \\
\hline A1 & 71.7 & $64.9-77.4$ & 56.9 & $47.5-65.3$ & \\
\hline B & 65.5 & $54.9-74.2$ & 49.9 & $37.8-60.9$ & \\
\hline Type of resection & & & & & 0.008 \\
\hline Minor & 80.0 & $75.3-83.8$ & 66.1 & $59.3-72.0$ & \\
\hline Major & 68.7 & $61.7-74.8$ & 57.2 & $48.6-64.9$ & \\
\hline Grade & & & & & $<0.001$ \\
\hline Well to moderate & 78.7 & $74.5-82.3$ & 67.1 & $61.2-72.3$ & \\
\hline Poor to undifferentiated & 61.8 & $52.1-70.1$ & 44.0 & $32.6-54.7$ & \\
\hline Lymphovascular invasion & & & & & $<0.001$ \\
\hline No & 84.1 & $79.5-87.7$ & 70.3 & $63.6-76.0$ & \\
\hline Yes & 62.7 & $55.8-68.8$ & 52.8 & $44.2-60.7$ & \\
\hline Liver capsule involvement & & & & & $<0.001$ \\
\hline No & 76.8 & $71.4-81.3$ & 64.7 & $58.0-70.7$ & \\
\hline Yes & 62.9 & $55.0-69.8$ & 52.5 & $43.5-60.7$ & \\
\hline Margin status & & & & & 0.004 \\
\hline R0 & 77.0 & $72.8-80.6$ & 63.7 & $58.1-68.8$ & \\
\hline $\mathrm{R} 1$ & 71.2 & $59.4-80.1$ & 59.5 & $42.6-73.0$ & \\
\hline
\end{tabular}


TABLE 2 continued

\begin{tabular}{llllll}
\hline Variable & 3-year OS $(\%)$ & $95 \%$ CI & 5-year OS (\%) & $95 \%$ CI & $p$ value \\
\hline R2 & 49.9 & $18.9-74.8$ & 24.9 & $1.6-62.7$ \\
\hline
\end{tabular}

$O S$ overall survival, $C I$ confidence interval, $I Q R$ interquartile range, ASA-PS American Society of Anesthesiologists performance score, $H B V$ hepatitis B virus, $H C V$ hepatitis $\mathrm{C}$ virus, $A F P \propto$-fetoprotein, $B C L C$ Barcelona Clinic Liver Cancer
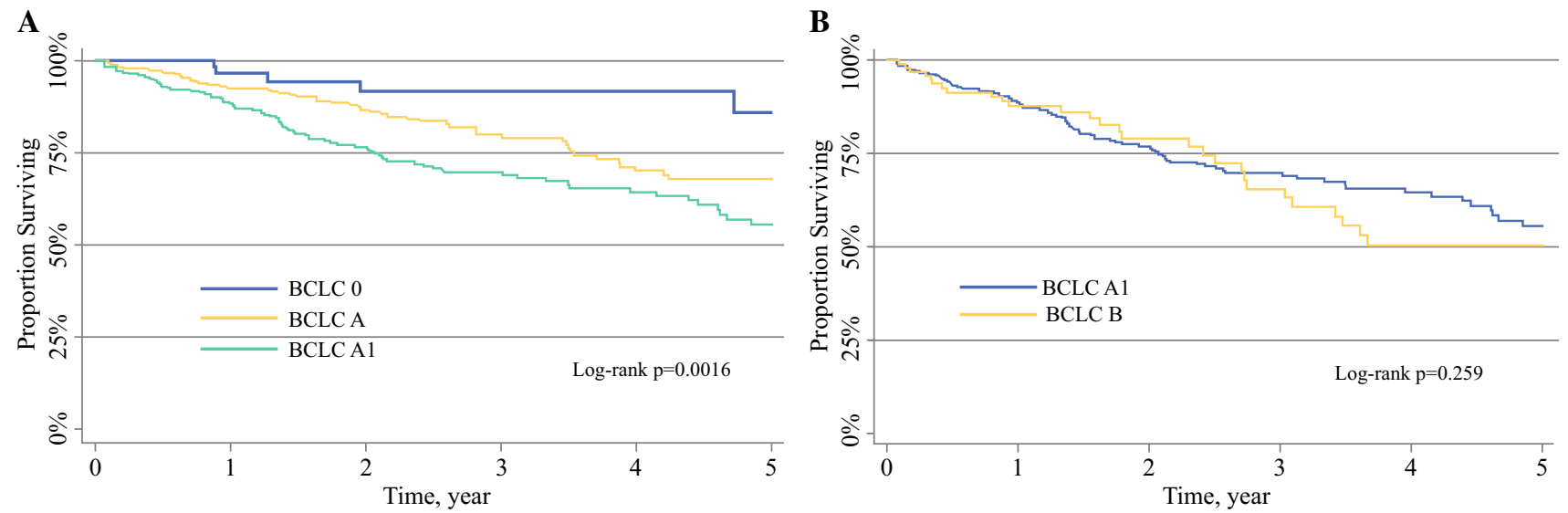

FIG. 2 Kaplan-Meier curves demonstrating differences in survival among patients with a BCLC stage 0, A, and A1 HCC, and b BCLC stage A1 versus stage B HCC. BCLC Barcelona Clinic Liver Cancer, $H C C$ hepatocellular carcinoma

TABLE 3 Multivariable analysis of survival

\begin{tabular}{lccc}
\hline Variable & HR & $95 \%$ CI & $p$ value \\
\hline AFP, ng/mL & & & \\
$\leq 400$ & Ref & & \\
$>400$ & 1.41 & $1.00-1.97$ & 0.047 \\
BCLC staging classification & & 0.005 \\
0 & 0.22 & $0.08-0.63$ & 0.043 \\
A & 0.62 & $0.39-0.98$ & 0.40 \\
A1 & 0.83 & $0.54-1.28$ & \\
B & Ref & & \\
Lymphovascular invasion & & \\
No & Ref & & \\
Yes & 1.83 & $1.32-2.55$ & \\
Margin status & & & 0.001 \\
R0 & Ref & & 0.031 \\
R1 & 0.81 & $0.51-1.29$ & \\
R2 & 2.36 & $1.08-5.17$ & \\
\hline
\end{tabular}

$\overline{A F P} \alpha$-fetoprotein, $B C L C$ Barcelona Clinic Liver Cancer, $H R$ hazard ratio, $C I$ confidence interval, $r$ f reference

ratio [HR] $0.22,95 \%$ CI $0.08-0.63 ; p=0.005)$ and $\mathrm{BCLC}$ stage A (HR 0.62, 95\% CI 0.39-0.98; $p=0.043$ ) HCC had $78 \%$ and $38 \%$ decreased hazards of death, respectively (Table 3). Nevertheless, after controlling for all measurable confounding factors, no difference in survival was noted among patients with BCLC stage A1 and B HCC
(HR 0.83, 95\% CI 0.54-1.28; $p=0.40$ ). In contrast, AFP $>400 \mathrm{ng} / \mathrm{mL} \quad(\mathrm{HR} \quad 1.41, \quad 95 \%$ CI $1.00-1.97$; $p=0.047)$, presence of microscopic lymphovascular invasion (HR 1.83, 95\% CI 1.32-2.55; $p<0.001$ ), and R2 margin status (HR 2.36, 95\% CI 1.08-5.17; $p=0.031$ ) were all associated with a higher hazard of death.

\section{Subgroup Analysis: Factors Associated with Overall Survival Among BCLC Stage Al and B HCC}

Among patients with BCLC stage A1 and B HCC, patients with AFP $\leq 400 \mathrm{ng} / \mathrm{mL}$ had a 5 -year OS of $60.0 \%$ (95\% CI 50.6-68.1) versus $44.5 \%$ (95\% CI 32.5-55.8) for individuals with AFP $>400 \mathrm{ng} / \mathrm{mL}(p<0.001)$ [Electronic Supplementary Table S1]. In addition, poorly/ undifferentiated tumor grade, presence of lymphovascular invasion, and $\mathrm{R} 1 / \mathrm{R} 2$ margin status were all associated with worse 5-year OS (all $p<0.001$ ).

\section{DISCUSSION}

Prognostic assessment is critical to construct the appropriate therapeutic strategy for patients diagnosed with HCC. ${ }^{4}$ The BCLC classification system not only assesses patient prognosis but also assigns treatment allocation based on prognostic subclasses. ${ }^{2,6}$ Although this system has been largely adopted in the West, there has recently been a growing skepticism as to whether the current BCLC 
classification performs well in terms of prognostic stratification, especially for patients with a single large tumor, which was previously considered BCLC stage B. ${ }^{1,13}$ In addition, a growing number of studies have advocated for liver resection outside the BCLC criteria, suggesting a shortcoming of the current stage-specific treatment proposed by the BCLC system. ${ }^{14-16}$ The current study was important because we specifically assessed the outcomes of patients undergoing surgery for HCC within (BCLC stage 0 and A) and outside (BCLC stage B) the BCLC criteria using an international multi-institutional database. Of note, among patients with very early- and early-stage HCC, patients with a single large tumor $(\geq 5 \mathrm{~cm}$; BCLC stage A1) had the worst OS. Perhaps of more interest, patients undergoing surgery for BCLC stage A1 HCC (within the BCLC criteria) had long-term outcomes comparable with patients undergoing surgery for BCLC stage B HCC (outside the BCLC criteria), even after controlling for all possible competing risk factors. These results indicate that while a single large tumor should be considered BCLC stage $\mathrm{A}$ in terms of treatment allocation (i.e. surgery), BCLC stage B is likely a better designation for these patients in terms of prognosis. In addition, these data support the notion that designation into BCLC stage B should not be considered an a priori contraindication to surgery, especially among patients with resectable tumors.

Previous studies have demonstrated that patients with HCC exceeding $5 \mathrm{~cm}$ have a distinct, worse prognosis compared with patients who have a smaller solitary tumor. ${ }^{11,15}$ Indeed, increasing tumor size has been associated with higher rates of microvascular invasion and more advanced histologic grade, both of which are known to increase recurrence rates. ${ }^{8,9}$ In fact, one previous study noted that the incidence of microvascular invasion nearly doubled among patients with tumors larger than $5 \mathrm{~cm}$ $(61 \%)$ compared with smaller tumors $(32 \%)$, and continued to increase among tumors larger than $10 \mathrm{~cm} .{ }^{9}$ In turn, patients with a solitary large tumor had higher recurrence rates and thus worse survival. ${ }^{9}$ Given these data, several investigators have suggested that these patients should be classified as BCLC stage B, rather than BCLC stage A. ${ }^{13,17}$ In the current study, among the 279 patients with a single large tumor, we noted that these patients had markedly worse prognosis compared with other patients who had early (BCLC stage A) or very early (BCLC stage 0) stage tumors (5-year OS: $56.9 \%$ vs. $69.0 \%$ vs. $86.2 \%$, respectively; $p=0.0016)$. Perhaps of more interest, the prognosis of these patients was similar to patients who had BCLC stage B tumors, even after controlling for all measured competing risk factors (HR 0.83, 95\% CI 0.54-1.28; $p=0.40)$. As such, data from the current study strongly suggest that patients with a single large HCC would be more appropriately classified as BCLC stage B in terms of prognosis.

While surgery for a single large $\mathrm{HCC}$ has been associated with acceptable long-term outcomes, ${ }^{14-16}$ the current BCLC classification recommends that patients with BCLC stage $\mathrm{B}$ should undergo $\mathrm{TACE}^{2}$. However, an increasing body of evidence has revealed that liver resection may be justified in select patients with intermediate (BCLC B) or even advanced (BCLC C) stage HCC. ${ }^{18-20}$ In a recent propensity score matched study, Kim et al. compared the outcomes of patients with BCLC stage B HCC following surgery $(n=80)$ versus no surgical treatment $(n=80) .^{18}$ The authors reported a 5-year OS of $63 \%$ among patients who underwent resection versus only $22 \%$ for patients in the non-surgical cohort. ${ }^{18}$ These data suggested that surgery can offer a survival benefit for potentially resectable BCLC stage B HCC. In a separate study, Wada et al. reported on patients with multinodular BCLC stage B HCC undergoing liver resection and demonstrated an acceptable 5-year OS of $63.4 \% .^{12}$ In addition, increasing evidence suggests that patients undergoing an anatomic resection may have improved outcomes compared with individuals who undergo a non-anatomic resection. ${ }^{2,21}$ Indeed, with an anatomic resection of the liver, Glisson pedicles are ligated and cut off in advance. ${ }^{22}$ In turn, microvascular invasive lesions, thought to be strongly correlated with disease relapse and more frequently noted in patients with single large tumors $(>5 \mathrm{~cm})$ or multifocal disease (i.e. BCLC A1 or BCLC B), may be more appropriately removed with anatomic resection of the liver provided that the patient has adequate liver remnant. ${ }^{21,22}$ The current study is one of the largest cohorts of patients undergoing liver resection outside the BCLC criteria ( $n=157$ patients with BCLC stage B HCC) reported in the literature. ${ }^{20,23,24}$ By exclusively analyzing BCLC stage B patients who underwent resection, we were able to determine that the 5-year OS of $49.9 \%$ (95\% CI 37.8-60.9) was similar to patients having BCLC stage A1 tumors. Thus, the data support surgery as being beneficial in select patients with BCLC stage B HCC. In addition, designation to BCLC stage B per se should not be considered a contraindication to surgery. Rather, preoperative AFP levels > $400 \mathrm{ng} / \mathrm{ml}$ were strongly associated with worse long-term outcomes and should be taken into consideration when planning surgery for patients outside the Barcelona criteria.

The results of the current study should be interpreted in the context of certain limitations. The retrospective nature of the study may have introduced some selection biases, (i.e. patients with BCLC stage B HCC had potentially resectable tumors and more favorable tumor biology), and as such the results should only be applied to select patients with HCC. In addition, the lack of a comparison group (i.e. 
TACE for BCLC stage B patients) did not allow us to draw definitive conclusions over the superiority of different treatment approaches in the management of patients.

\section{CONCLUSIONS}

The prognosis of patients with a single large HCC was worse compared with other BCLC stage A patients, but was similar to patients presenting with BCLC stage B tumors following liver resection. Surgery provided acceptable long-term outcomes among select patients with BCLC stage B HCC. Designation into BCLC stage B should not be considered an a priori contraindication to surgery among patients with resectable tumors. While patients with a single large HCC should be treated as BCLC A patients, their prognosis was more akin to BCLC $\mathrm{B}$ patients. This point further highlights that the confluence of staging and treatment allocation characterized in the BCLC staging system may represent an oversimplification of how to care for patients with HCC.

DISCLOSURE The authors declare that they have no conflict of interest.

\section{REFERENCES}

1. Beal EW, Tumin D, Kabir A, et al. Trends in the mortality of hepatocellular carcinoma in the United States. J Gastrointest Surg. 2017;21(12):2033-2038.

2. European Association for the Study of the Liver. EASL Clinical practice guidelines: management of hepatocellular carcinoma. $J$ Hepatol. 2018;69(1):182-236.

3. Marrero JA, Kulik LM, Sirlin CB, et al. Diagnosis, staging, and management of hepatocellular carcinoma: 2018 practice guidance by the American Association for the Study of Liver Diseases. Hepatology. 2018;68(2):723-750.

4. Marrero JA, Fontana RJ, Barrat A, et al. Prognosis of hepatocellular carcinoma: comparison of 7 staging systems in an American cohort. Hepatology. 2005;41(4):707-716.

5. O'Neil BH, Venook AP. Hepatocellular carcinoma: the role of the North American GI Steering Committee Hepatobiliary Task Force and the advent of effective drug therapy. Oncologist. 2007;12(12):1425-1432.

6. Cillo U, Vitale A, Grigoletto F, et al. Prospective validation of the Barcelona Clinic Liver Cancer staging system. J Hepatol. 2006;44(4):723-731.

7. European Association for the Study of the Liver, European Organisation for Research and Treatment of Cancer. EASLEORTC clinical practice guidelines: management of hepatocellular carcinoma. J Hepatol. 2012;56(4):908-943.

8. Esnaola NF, Lauwers GY, Mirza NQ, et al. Predictors of microvascular invasion in patients with hepatocellular carcinoma who are candidates for orthotopic liver transplantation. $J$ Gastrointest Surg. 2002;6(2):224-232 (discussion 232).
9. Pawlik TM, Delman KA, Vauthey JN, et al. Tumor size predicts vascular invasion and histologic grade: implications for selection of surgical treatment for hepatocellular carcinoma. Liver Transpl. 2005;11(9):1086-1092.

10. Guo H, Wu T, Lu Q, et al. Surgical resection improves long-term survival of patients with hepatocellular carcinoma across different Barcelona Clinic Liver Cancer stages. Cancer Manag Res. 2018;10:361-369.

11. Cho Y, Sinn DH, Yu SJ, et al. Survival analysis of single large $(>5 \mathrm{~cm})$ hepatocellular carcinoma patients: BCLC A versus B. PLoS One. 2016;11(11):e0165722.

12. Wada H, Eguchi $\mathrm{H}$, Noda $\mathrm{T}$, et al. Selection criteria for hepatic resection in intermediate-stage (BCLC stage B) multiple hepatocellular carcinoma. Surgery. 2016;160(5):1227-1235.

13. Jung YK, Jung CH, Seo YS, et al. BCLC stage B is a better designation for single large hepatocellular carcinoma than BCLC stage A. J Gastroenterol Hepatol. 2016;31(2):467-474.

14. $\mathrm{Ng} \mathrm{KK}$, Vauthey JN, Pawlik TM, et al. Is hepatic resection for large or multinodular hepatocellular carcinoma justified? Results from a multi-institutional database. Ann Surg Oncol. 2005;12(5):364-373.

15. Pawlik TM, Poon RT, Abdalla EK, et al. Critical appraisal of the clinical and pathologic predictors of survival after resection of large hepatocellular carcinoma. Arch Surg. 2005;140(5):450-457 (discussion 457-458).

16. Pandey D, Lee KH, Wai CT, Wagholikar G, Tan KC. Long term outcome and prognostic factors for large hepatocellular carcinoma (10 cm or more) after surgical resection. Ann Surg Oncol. 2007;14(10):2817-2823.

17. Liu PH, Su CW, Hsu CY, et al. Solitary large hepatocellular carcinoma: staging and treatment strategy. PLoS One. 2016;11(5):e0155588.

18. Kim H, Ahn SW, Hong SK, et al. Survival benefit of liver resection for Barcelona Clinic Liver Cancer stage B hepatocellular carcinoma. Br J Surg. 2017;104(8):1045-1052.

19. Liang L, Xing $H$, Zhang $H$, et al. Surgical resection versus transarterial chemoembolization for BCLC intermediate stage hepatocellular carcinoma: a systematic review and meta-analysis. HPB (Oxford). 2018;20(2):110-119.

20. Hyun MH, Lee YS, Kim JH, et al. Hepatic resection compared to chemoembolization in intermediate- to advanced-stage hepatocellular carcinoma: a meta-analysis of high-quality studies. Hepatology. 2018;68(3):977-993.

21. Moris D, Tsilimigras DI, Kostakis ID, et al. Anatomic versus non-anatomic resection for hepatocellular carcinoma: a systematic review and meta-analysis. Eur J Surg Oncol. 2018;44(7):927938.

22. Moris D, Rahnemai-Azar AA, Tsilimigras DI, et al. Updates and critical insights on glissonian approach in liver surgery. J Gastrointest Surg. 2018;22(1):154-163.

23. Labgaa I, Demartines N, Melloul E. Surgical resection versus transarterial chemoembolization for intermediate stage hepatocellular carcinoma (BCLC-B): an unsolved question. Hepatology. 2019;69(2):923.

24. Mo DC, Jia RR, Zhong JH. hepatic resection compared to chemoembolization in intermediate to advanced-stage HCC: a comment for moving forward. Hepatology. Epub 5 Dec 2018.

Publisher's Note Springer Nature remains neutral with regard to jurisdictional claims in published maps and institutional affiliations. 